\title{
Advances of Carbon-Based Materials for Lithium Metal Anodes
}

\author{
Kaikai Tang ${ }^{1}$, Jun Xiao ${ }^{1}$, Xiao $\mathrm{Li}^{1}$, Dandan Wang ${ }^{1}$, Mengqi Long ${ }^{1}$, Jun Chen ${ }^{1}$, \\ Hong Gao ${ }^{1,2 *}$, Weihua Chen ${ }^{3}$, Chuntai Liu ${ }^{3}$ and Hao Liu ${ }^{1,4 *}$ \\ 1 Joint International Laboratory on Environmental and Energy Frontier Materials, School of Environmental and Chemical \\ Engineering, Shanghai University, Shanghai, China, ${ }^{2}$ State Key Laboratory of Advanced Special Steel, Shanghai Key \\ Laboratory of Advanced Ferrometallurgy, Shanghai University, Shanghai, China, ${ }^{3}$ Key Laboratory of Materials Processing and \\ Mold (Zhengzhou University), Ministry of Education, Zhengzhou, China, ${ }^{4}$ Centre for Clean Energy Technology, Faculty of \\ Science, School of Mathematical and Physical Sciences, University of Technology Sydney, Sydney, NSW, Australia
}

Lithium metal with high theoretical specific capacity $\left(3,860 \mathrm{mAh} \mathrm{g}^{-1}\right)$, low mass density, and low electrochemical potential (-3. $040 \mathrm{~V}$ vs. SHE) is an ideal candidate of the battery anode. However, the challenges including dendrite propagation, volume fluctuation, and unstable solid electrolyte interphase of lithium metal during the lithium plating impede the practical development of Lithium metal batteries (LMBs). Carbon-based materials with diverse structures and functions are ideal candidates to address the challenges in LMBs. Herein, we briefly summarize the main challenges as well as the recent achievements of lithium metal anode in terms of utilizing carbon-based materials as electrolyte additives, current collectors and composite anodes. Meanwhile, we propose the critical challenges that need to be addressed and perspectives for ways forward to boost the advancement of LMBs.

Keywords: composite anodes, current collectors, additives, carbon-based materials, lithium metal anodes, battereis

\section{INTRODUCTION}

Since Sony commercialized lithium-ion batteries (LIBs) in 1991, rechargeable LIBs have been successfully applied to portable electronics and electric vehicles (Yang G. et al., 2019; Yang T. et al., 2019; Lee et al., 2020; Maroufi et al., 2020; Pellow et al., 2020). However, the constrained energy density of the traditional LIBs based on intercalation chemistry are unable to meet the ever-growing requirement of high-energy-density batteries. Lithium metal batteries (LMBs) with high capacity and high energy density have attracted numerous attentions. Compared with commercial LIBs, LMBs employ metallic $\mathrm{Li}$ as an anode, which is based on a continuous plating/stripping mechanism, contributing to higher energy output (Chen C. et al., 2019; Zhang T. et al., 2019; Widijatmoko et al., 2020; Ye et al., 2020). Lithium metal anodes are known as the "Holy Grail" electrodes due to the unique advantages, such as the lowest density among metals, high theoretical specific capacity $\left(3,860 \mathrm{mAh}^{-1}\right)$ and the lowest electrochemical potential ( $-3.040 \mathrm{~V}$ vs. standard hydrogen electrode) (Xie et al., 2019; Shi et al., 2020; Zhang Q. et al., 2020; Zhou Y. et al., 2020). Moreover, when LMBs collocate with high capacity cathodes, such as sulfur $(\mathrm{S})$ and oxygen $\left(\mathrm{O}_{2}\right)$, it can achieve excellent specific energy and be regarded as promising next-generation energy storage systems beyond LIBs and other storage systems (Hong et al., 2019; Sloop et al., 2019; Xiao et al., 2019; Gan et al., 2020; Guo et al., 2020; Li W. T. et al., 2020). Unfortunately, the development of LMBs is hindered by the inevitable shortcomings of lithium metal anode, including dendrite propagation, volume fluctuation, and unstable solid electrolyte interphase (SEI), originating from the high chemical 
reactivity and "hostless" nature of lithium metal during the plating/stripping process (Li Q. et al., 2018; Li Z. et al., 2020; Pan et al., 2020).

Carbon-based materials with good electrical and thermal conductivity, excellent structural stability, as well as high surface area and abundant surface functional groups have been extensively applied in numerous research fields (Liu et al., 2017; Yan et al., 2019; Tian et al., 2020). Even more noteworthy is that carbon-based materials offer a versatile platform to construct new architectures with interconnected porosity and active sites (Ge et al., 2018; Zhang Z. et al., 2018a; Zhang W. et al., 2019). The surface chemistry of carbon-based materials can be easily modified to improve the binding properties. Consequently, doping or co-doping with different heteroatoms (N, S, P) (Wei et al., 2019; Liu et al., 2020; Ma et al., 2020; Zhang D. M. et al., 2020) makes the carbon-based materials exhibit further enhancement of the electrocatalytic activity. Owing to these diversified functions of carbon-based materials, they have been intensively used to ameliorate the challenges of LMBs (Zuo et al., 2018; Xue et al., 2019; Huang et al., 2020).

Although there are many reported reviews on LMBs, very few reports that specifically describe carbon-based materials in addressing the challenges in LMBs. This review provides a comprehensive overview of the application of carbon-based materials in improving the performance of lithium metal anodes. In this review, we focus on the main challenges faced by lithium metal anodes and emphasize the effective strategies employing carbon-based materials as electrolyte additives, current collectors and composite anodes to achieve high performance lithium metal anodes. We believe that this review is informative and could offer some new insights for this exciting area.

\section{CHALLENGES OF LITHIUM METAL ANODES}

The LMBs were born in the 1970's. However, they have almost stagnated since then, lagging far behind the development of LIBs. As we all know, lithium metal is very active and can react with almost all organic electrolytes to form a SEI (Figure 1). An ideal SEI film should be electrically insulating and ionically conductive. Moreover, it should have good chemical and mechanical stability to withstand the volume change and protect the lithium metal from further exposure to the electrolyte. Nevertheless, due to the "hostless" property and unevenness of lithium metal surface, $\mathrm{Li}^{+}$ preferentially deposits at the tips, resulting in uneven deposition of lithium and the formation of dendrites during the repeatedly stripping and plating process. The dendrites inevitably cause the electrode volume changes, which lead to the destruction of SEI film and then exposure of fresh lithium metal to the electrolyte. This is followed by continuous side reactions until the electrolyte is depleted, which have a negative impact on the Coulomb efficiency (CE). At the same time, the aforementioned process can in turn exacerbate the dendrite growth.

Eventually, there are two possibilities of the uncontrolled dendrites. Firstly, the dendrites may fall off from the anode surface to form "dead Li", causing the loss of active materials

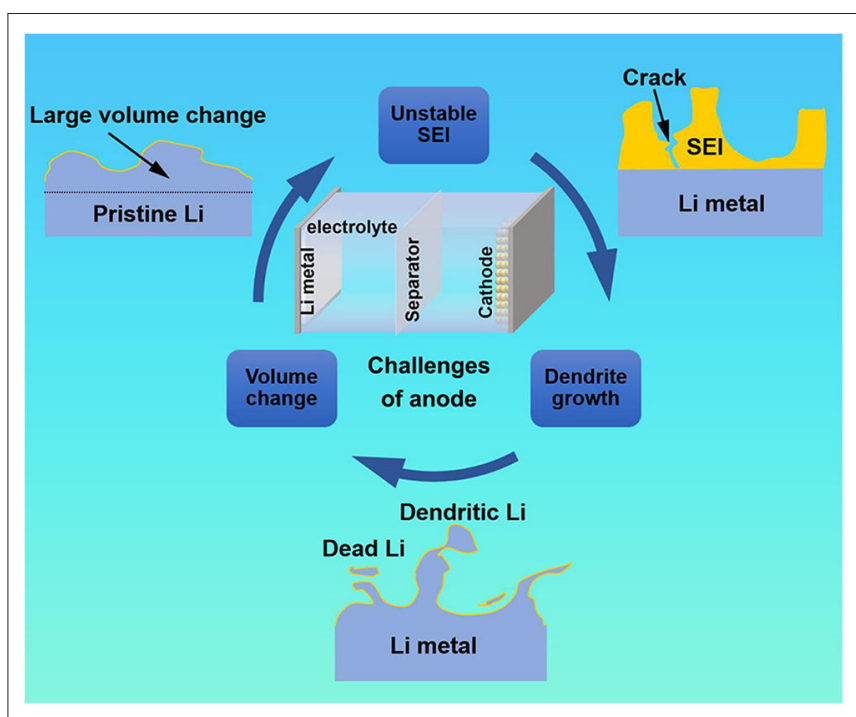

FIGURE 1 | Schematic diagram of the challenge of anode for LMBs.

and greatly reduce the utilization of Li. Secondly, the dendrites may pierce the separator to reach the cathode, causing a short circuit. These intrinsic challenges of lithium metal lead to many undesirable drawbacks such as unstable SEI, severe volume change and uncontrolled dendrite growth during electroplating/stripping in the rechargeable LMBs, which have been hindered their practical applications over the past 40 years (Zhang R. et al., 2017).

\section{APPLICATION OF CARBON-BASED MATERIALS IN LITHIUM METAL ANODES}

So far, tremendous strategies have been proposed to suppress the dendrites growth. These strategies can be classified into the following categories: (1) electrolyte manipulation, including modification of additives in the electrolyte (Zhang X. Q. et al., 2017; Huang et al., 2018; Li X. et al., 2018), and adoption of solid or polymer electrolytes (Aldalur et al., 2018; Girard et al., 2019; Huo et al., 2020; Hu Z. et al., 2020); (2) SEI engineering (Li C. et al., 2019; Yan et al., 2020), such as artificial SEI film (Zhu et al., 2019); (3) electrode structure design (Zhang Y. et al., 2018, 2019).

Carbon-based materials with unique physical and chemical properties can act as electrolyte additives to inhibit the dendrite growth. In addition, the various structures, high surface area and flexibility enable the carbon-based materials to be as hosts to accommodate the volume change (Jorge et al., 2019; Nan et al., 2019; Zhang X. et al., 2020).

\section{Carbon-Based Materials to Construct Composite Anodes}

On account of the intrinsic "hostless" of lithium metal, the lithium deposits on the planar electrode will undergo severe volume changes, resulting in continuous fracture and regeneration of the SEI film. Unstable SEI film will seriously 
reduce the lifespan of the battery. Therefore, confining the lithium metal in scaffold is an effective way to accommodate the volume change (Yang et al., 2018; Pei et al., 2019; Tang et al., 2020). The as-prepared structured composite anode can regulate the $\mathrm{Li}^{+}$deposition and induce dendrite-free morphology, thereby achieving a high capacity and long cycle life lithium metal anode (Wang et al., 2017; Zhang R. et al., 2018a). The 3D porous scaffold with multiple ion and electron transfer paths and high surface area is often employed as host for lithium metal. Moreover, the current density can be dissipated by this $3 \mathrm{D}$ conductive porous scaffolds, resulting in a lower local current density (Chazalviel, 1990; Zhou T. et al., 2020), which can effectively control the growth rates of lithium dendrites.

The 3D porous scaffolds, such as copper foam (Yang et al., 2018; Yue et al., 2019b), nickel foam (Huang X. et al., 2019; Sun et al., 2019), mesoporous carbon (Zhang S. J. et al., 2019; Jeong et al., 2020), and carbon nanotube sponge (Yang G. et al., 2019), can accommodate infinite volume expansion and suppress dendrite growth during repeated electroplating/stripping processes. Besides, the composite electrode, assembled with a carbon-based skeleton, has good flexibility and can be used on wearable electronic devices. Nevertheless, most hosts need to be coated with a lithiophilic layer because of their poor lithiophilicity and the requirements of constructing a composite anode. Unlike metal frameworks that require complex processes to enhance lithiophilicity, carbon-based scaffolds have very significant advantages in practical applications. For example, some lithiophilic functional groups can be introduced into the most carbon hosts by facile surface chemistry approaches. Additionally, carbon-based materials with low density enable the high practical energy density batteries.

Tao et al. reported a simple surface ozonolysis and ammoniation treatment strategy to tune the lipophilicity of carbon scaffold (Tao et al., 2020). The flexible and lithiophilic carbon film (CF) is composed of multiple layered interwoven nanofibers. Due to the good mechanical strength and thermal stability, CF can be easily wetted by molten lithium to form a stable Li@CF composite anode. The as-prepared Li@CF composite electrode could deliver a high practical capacity of $3,222 \mathrm{mAh}^{-1}$ and behave a good rate performance. In addition, no obvious dendrites have been observed in the electrode.

Lithiophilic coating is an effective strategy to enhance the wettability between liquefied lithium and carbon matrix, because these coating layers can react with molten lithium to reduce the surface energy of carbon matrix or form alloys with lithium. Silicon can coat on the carbon-based materials via chemical vapor deposition to form the $\mathrm{Li}_{\mathrm{X}} \mathrm{Si}$ alloys (Liang et al., 2016; Hapuarachchi et al., 2018). Thus, the molten metallic lithium can easily and quickly flow into the scaffolds with silicon coating. Some metal oxides, such as $\mathrm{ZnO}$ (Zhao et al., 2019; Yue et al., 2020), $\mathrm{CuO}$ (Wu et al., 2018; Zhang C. et al., 2018; Huang K. et al., 2019), $\mathrm{Co}_{3} \mathrm{O}_{4}$ (Li S. Y. et al., 2019; Pan et al., 2019), can undergo redox reactions with molten lithium. Based on this fact, the scaffold coated with metal oxide can obtain enhanced lithiophilicity. Yue et al. coated $\mathrm{Cu}_{\mathrm{x}} \mathrm{O}$ $(\mathrm{CFeltCu})$ on a porous carbon felt (CFelt) and prepared a stable

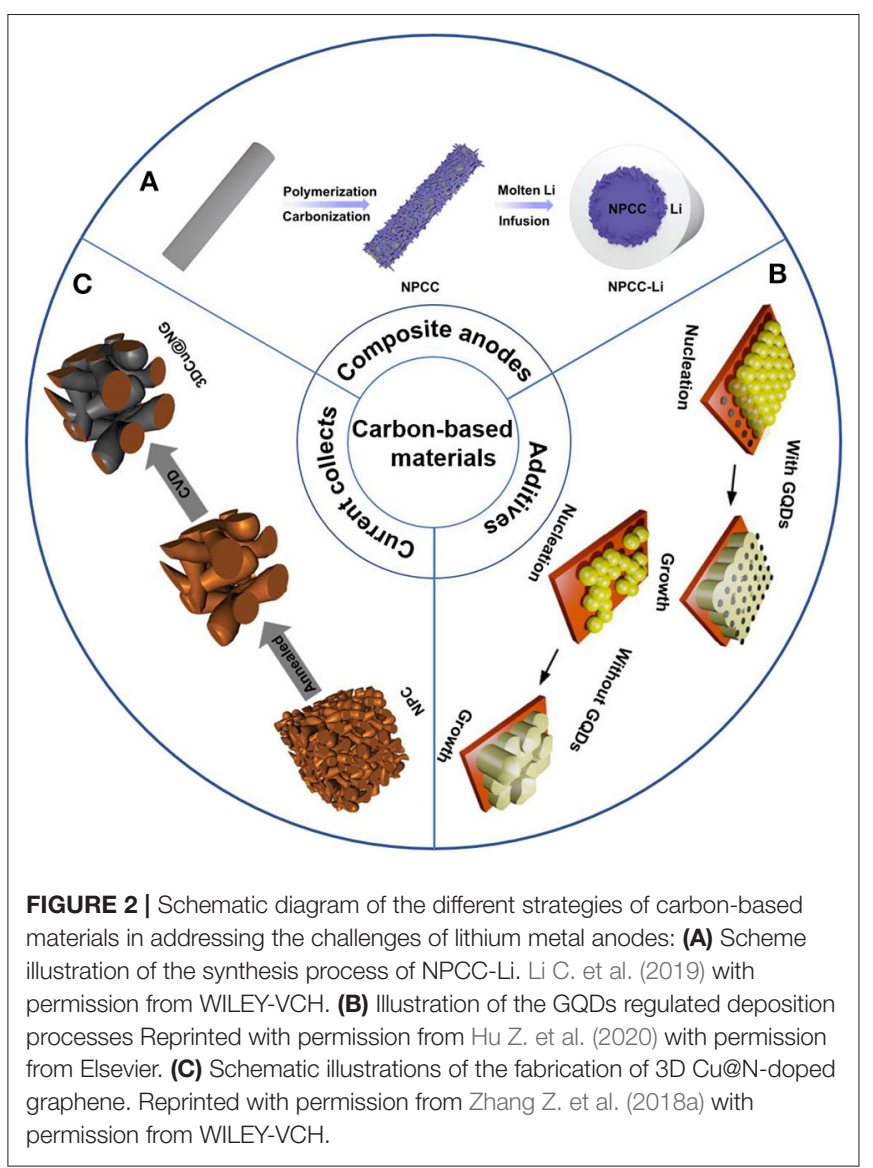

CFeltCu-Li composite anode via thermal infusion method (Yue et al., 2019a). During the thermal infusion process, the $\mathrm{Cu}$ nanoparticles derived from copper oxide reduced by molten lithium are evenly dispersed on the surface of the CFelt. To some extent, these $\mathrm{Cu}$ nanoparticles with high conductivity can not only regulate the Li stripping/plating behavior, but also reduce the local current density of anode. Therefore, the CFeltCuLi composite anode presented outstanding cycle stability (over $1,000 \mathrm{~h})$ and low overpotential $(25 \mathrm{mV})$ without dendrite growth in symmetric cells.

Although the metal oxide coating can significantly improve the lithium affinity of carbon-based materials, the specific capacity and rate performance of lithium anodes have deteriorated to some extent. Carbon matrix with heteroatom co-doping can effectively overcome the above mentioned obstacle. A stable lithium composite anode that is composed of $\mathrm{N}$ and $\mathrm{P}$ co-doped carbon cloth and lithium metal, was presented by Li and coworkers (Figure 2A) (Li K. et al., 2019). N and P can provide enhanced surface lithiopholicity for carbon-based material, facilitating molten lithium diffusion and uniform coating. This composite anode delivered stable voltage hysteresis over $600 \mathrm{~h}$ at a current density of $3 \mathrm{~mA} \mathrm{~cm}^{-2}$.

All the aforesaid approaches require complex preparation processes. In order to simplify the synthesis process, Go et al. made carbon cloth more affinity between lithium and carbon 
by a facile heat treatment in air (Go et al., 2019). Numerous nanocrevasses can be introduced in carbon cloth during the heat treatment, allowing the successful infusion of molten lithium. As a result, the as-prepared composite anode with lower local current density presents long-term cycling and dendritefree morphology.

\section{Carbon-Based Materials as Additive}

Liquid organic electrolytes play a vital role in LIBs, due to their good wettability with electrode and ionic conductivity. However, the side reaction between lithium metal and electrolyte has negative affect on the electrochemical performance of lithium metal anode. The liquid electrolyte is composed of solvent, lithium salt and additives, which determine the uniformity and stability of SEI film. Therefore, modifying the additives can improve the performance of the electrolyte, then changing the deposition morphology of lithium (Tao et al., 2017; Wang et al., 2020).

In general, soluble Li-containing compounds and organic compounds, such as $\mathrm{LiNO}_{3}$ (Yan et al., 2018), LiF (Wang et al., 2019) and fluoroethylene carbonate (FEC) (Zhang R. et al., 2017), are commonly employed as additives. These additives are generally used as sacrifices to react with lithium metal in advance, forming a SEI film with controllable composition and good stability. On the contrary, when the carbon-based material is used as additive, it serves as the initial nucleation site for Li deposition instead of reacting with Li. Nanodiamond with a size of $\sim 5 \mathrm{~nm}$ and high crystallinity is an early member of the carbon-based material family (Cheng et al., 2017). The nanodiamond particles treated by octadecylamine (ODA) can be well-dispersed in the ester-based electrolyte. In $\mathrm{Li} \| \mathrm{Cu}$ halfcell batteries, due to the nanodiamonds inherit large surface area and strong binding energy with $\mathrm{Li}$, the $\mathrm{Li}^{+}$can adsorb on the surface as the initial heterogeneous seeds instead of growing on the copper current collector. Moreover, nanodiamond-guided Li deposits are small enough to form a uniform distribution of deposition. When charging, the co-deposits of nanodiamond and $\mathrm{Li}$ can be stripped into the electrolyte to maintain a stable content of nanodiamond in the electrolyte, improving the cycling stability of the Li anode. Whereas, the nanodiamonds tend to aggregate and form clusters easily, which is negatively affect the long-term cycle.

With the development of technology, more and more new materials are recognized and applied in various fields. Graphene quantum dots (GQDs) with tiny size can be well-dispersed in the electrolyte without further modification (Deng et al., 2016; Park et al., 2016; Tam et al., 2019). Hu et al. directly added GQDs to the electrolyte, which is composed of Li bis (trifluoromethane)-sulfonimide (LiTFSI, $1.0 \mathrm{M}$ ), $2.0 \mathrm{wt} \% \mathrm{LiNO}_{3}$, 1,3-dioxolane (DOL) and 1,2-dimethoxyethane (DME; 1:1 by volume) (Hu Y. et al., 2020). Due to the quantum confinement effect (Zhang W. et al., 2018), GQDs act as the heterogeneous seeds enable continuously adjust ion dispersion and avoid high local electric field in the subsequent plating process, which is conducive to the uniform deposition and inhibit the dendrite growth (Figure 2B). However, the complex synthesis procedures and extreme conditions of GQDs leads to high costs, which limits its large-scale application.

\section{Carbon-Based Materials as Current Collect}

The current collector is one of the important components of LIBs. It is commonly used as a substrate to support active materials. During battery operation, the current collector not only transfer electrons between the active material and the external circuit, but also diffuse the heat generated inside the battery (Jin et al., 2018; Zhang Z. et al., 2018b). Generally, planar copper foil is used as anode current collector, while it is easy to cause severe dendrite formation. Due to the porosity, low cost, good electronic conductivity and confinement of $3 \mathrm{D}$ current collectors, they have attracted great attention from researchers. 3D metallic materials, such as copper foam, nickel foam and aluminum foam, with good electronic conductivity and high specific surface area have been regarded as the most competitive candidates. However, the nucleation overpotential of lithium deposited on the 3D metal structure is relatively higher than other current collectors, causing uneven nucleation and inhomogeneous lithium deposition. Carbon-based materials have good lithium affinity, and composite with metal materials can effectively improve battery performance. When N-doped graphene combined with $3 \mathrm{D}$ metal materials, an improving LMBs performance can be achieved. Zhang et al. prepared a $3 \mathrm{D}$ porous copper coated with $\mathrm{N}$-doped graphene via a CVD process (Figure 2C) (Zhang R. et al., 2018b). Due to the presence of pyridinic and pyrrolic nitrogen, there is a strong interaction between $\mathrm{N}$-doped graphene and $\mathrm{Li}^{+}$, leading to homogeneous $\mathrm{Li}^{+}$flux and a uniform Li deposition.

The morphology of carbon-based material is critical to control the lithium loading and deposition location. The 3D carbonbased materials with random sponge-like structure lead to $\mathrm{Li}^{+}$ preferentially deposit on the outer surface of the $3 \mathrm{D}$ framework. To solve this problem, a sequence of materials with regular structures have been investigated. A 3D construction is fabricated by vertically arranged nanofibers (VACNFs) directly grown on a planar copper foil. When it acts as a host for the lithium metal anode (Chen Y. et al., 2019), the special structure of VACNFs not only provides well-aligned brush-like space for $\mathrm{Li}^{+}$deposition, but also enhances the surface electrochemical activity because of the active graphitic edge sites. Therefore, this composite material can effectively decrease the local current density, suppress the lithium dendrite growth and result in dendrite-free Li deposits.

Although the 3D metal structure exhibits excellent performance as a current collector, the defects, such as high density and easy erosion, cannot meet the requirements of high-performance storage systems. Compared with active materials, the density of metal current collectors is generally higher, resulting in a low mass proportion of active materials in the entire electrode, which inhibits the improvement of the energy density of the entire battery (Zhou et al., 2018). During the repeated charge/discharge process, the metal current collector electrochemically eroded, resulting in a short cycle life. Carbon-based materials with light weight and good chemical stability can avoid the aforementioned problems encountered by metal current collectors. Among carbon-based materials, carbon 
nanotubes (CNTs) are highly conductive and commercially available, and as an electrode behaving good lithium storage capacity (Che et al., 1998). Yang et al. used the commercial CNTs sponge as a current collector, which has high specific surface area and graphitic-amorphous carbon composite feature (Yang G. et al., 2019). In the initial stage of electroplating, the lithiumstorage of the CNTs (above $0 \mathrm{~V}$ ) makes it a "pre-lithiated" host, enhancing its wettability with subsequent lithium deposits (below $0 \mathrm{~V}$ ) and lowering the lithium nucleation overpotential. More importantly, the high surface area of the porous CNTs sponge enable the increased density of lithium nucleation sites and reduced local current density on the carbon nanotubes as well as uniform lithium deposition.

\section{CONCLUSIONS}

In recent decades, researchers have been committed to developing more strategies to meet the safety and high energy storage requirements of lithium metal anodes. Carbon-based materials with various structures and unique chemical properties play a significant role in minimizing the shortcomings of lithium metal anodes. This review outlines the challenges of lithium metal anodes and the diverse strategies of carbon-based materials in advanced LMBs.

The diversity of carbon-based materials makes it play a specific role in different strategies to solve the safety issues of lithium metal anodes. Nanodiamonds and GQDs with a size of several nanometers can be used as electrolyte additives to form initial nucleation sites, guiding the uniform deposition of $\mathrm{Li}^{+}$on the electrode surface. As the size of the carbon-based material increases, it has greater flexibility, higher porosity and a larger surface area, which helps it be modified or composited with other materials. The abundant functional groups on the surface of the carbon-based material enable it combine with other metal oxides or as a coating material for other $3 \mathrm{D}$ porous frameworks. The 3D porous scaffolds composite constructed with lithiophilic materials can be used as a current collector for $\mathrm{Li}^{+}$deposition and be assembled with lithium metal to form a composite electrode.

\section{REFERENCES}

Aldalur, I., Martinez-Ibanez, M., Piszcz, M., Zhang, H., and Armanda, M. (2018). Self-standing highly conductive solid electrolytes based on block copolymers for rechargeable all-solid-state lithium-metal batteries. Batteries Supercaps 1, 149-159. doi: 10.1002/batt.201800048

Chazalviel, J. (1990). Electrochemical aspects of the generation of ramified metallic electrodeposits. Phys. Rev. A 42, 7355-7367. doi: 10.1103/physreva.42.7355

Che, G., Lakshmi, B. B., Fisher, E. R., and Martin, C. R. (1998). Carbon nanotubule membranes for electrochemical energy storage and production. Nature 393, 346-349. doi: 10.1038/30694

Chen, C., Ma, Y., and Wang, C. (2019). Investigation of electrochemical performance of montmorillonite clay as Li-ion battery electrode. Sustain. Mater. Techno. 19:e00086. doi: 10.1016/j.susmat.2018.e00086

Chen, Y., Elangovan, A., Zeng, D., Zhang, Y., Ke, H., Li, J., et al. (2019). Vertically aligned carbon nanofibers on $\mathrm{Cu}$ foil as a $3 \mathrm{D}$ current collector for reversible $\mathrm{Li}$ plating/stripping toward high-performance Li-S batteries. Adv. Funct. Mater. 30:1906444. doi: 10.1002/adfm.201906444
Therefore, the 3D porous scaffolds can effectively overcome the "hostless" problem of lithium metal, accommodate huge electrode volume changes during electrochemical process, as well as contribute to a stable cycle life.

On account of the special structural and morphological features, carbon-based materials are also widely used in other research fields. Carbon-based materials can not only be employed as the electrode materials for LIBs and supercapacitors, but also act as the metal-free electrocatalysts for oxygen reduction reaction, oxygen evolution reaction, and hydrogen evolution reaction, because of its large specific surface area, defective sites, as well as tunable electronic structure.

Carbon-based materials with low price, abundant nature reserves, versatile structure easy fabrication, have a significant impact in the field of electrocatalysis and energy storage, especially for large-scale high-energy-density batteries. It is believed that the development of carbon-based materials plays a significant role in the commercial application of LMBs.

\section{AUTHOR CONTRIBUTIONS}

All authors listed have made a substantial, direct and intellectual contribution to the work, and approved it for publication.

\section{FUNDING}

This project was financially supported by the Australian Research Council (ARC) through the ARC Discovery project (DP180102297) and Future Fellowship (FT180100705). HG and HL thank the Open Project of State Key Laboratory of Advanced Special Steel, Shanghai Key Laboratory of Advanced Ferrometallurgy and the Science and Technology Commission of Shanghai Municipality (No. 19DZ2270200). HG appreciates the support from the China Postdoctoral Science Foundation Funded Project (2019M661464). All authors thank the support from Joint International Laboratory on Environmental and Energy Frontier Materials and Innovation Research Team of High-Level Local Universities in Shanghai.

Cheng, X. B., Zhao, M. Q., Chen, C., Pentecost, A., Maleski, K., Mathis, T., et al. (2017). Nanodiamonds suppress the growth of lithium dendrites. Nat. Commun. 8:336. doi: 10.1038/s41467-017-00519-2

Deng, G., Chao, D. L., Guo, Y. W., Chen, Z., Wang, H. H., Savilov, S. V., et al. (2016). Graphene quantum dots-shielded $\mathrm{Na}_{3}(\mathrm{VO})_{2}\left(\mathrm{PO}_{4}\right)_{2} \mathrm{~F} @ \mathrm{C}$ nanocuboids as robust cathode for Na-ion battery. Energy Storage Materials 5, 198-204. doi: 10.1016/j.ensm.2016.07.007

Gan, B. L., Tang, K. K., Chen, Y. L., Wang, D. D., Wang, N., Li, W. X., et al. (2020). Concrete-like high sulfur content cathodes with enhanced electrochemical performance for lithium-sulfur batteries. J. Energy Chem. 42, 174-179. doi: 10.1016/j.jechem.2019.06.003

Ge, P., Hou, H. S., Li, S. J., Yang, L., and Ji, X. B. (2018). Tailoring rod-like FeSe $\mathrm{S}_{2}$ coated with nitrogen-doped carbon for high-performance sodium storage. $A d v$. Funct. Mater. 28:1801765. doi: 10.1002/adfm.201801765

Girard, G. M. A., Wang, X., Yunis, R., MacFarlane, D. R., Bhattacharyya, A. J., Forsyth, M., et al. (2019). Sustainable, dendrite free lithium-metal electrode cycling achieved with polymer composite electrolytes based on a poly(ionic liquid) host. Batteries Supercaps 2, 229-239. doi: 10.1002/batt.201800120 
Go, W., Kim, M. H., Park, J., Lim, C. H., Joo, S. H., Kim, Y., et al. (2019). Nanocrevasse-rich carbon fibers for stable lithium and sodium metal anodes. Nano Lett. 19, 1504-1511. doi: 10.1021/acs.nanolett.8b04106

Guo, X., Zhang, W. X., Zhang, J. Q., Zhou, D., Tang, X., Xu, X. F., et al. (2020). Boosting sodium storage in two-dimensional phosphorene/ $\mathrm{Ti}_{3} \mathrm{C}_{2} \mathrm{~T}_{\mathrm{X}}$ MXene nanoarchitectures with stable fluorinated interphase. ACS Nano 14, 3651-3659. doi: 10.1021/acsnano.0c00177

Hapuarachchi, S. N. S., Nerkar, J. Y., Wasalathilake, K. C., Chen, H., Zhang, S., O'Mullane, A. P., et al. (2018). Utilizing room temperature liquid metals for mechanically robust silicon anodes in lithium-ion batteries. Batteries Supercaps 1, 122-128. doi: 10.1002/batt.201800047

Hong, Y. S., Zhao, C. Z., Xiao, Y., Xu, R., Xu, J. J., Huang, J. Q., et al. (2019). Safe lithium-metal anodes for $\mathrm{Li}_{-} \mathrm{O}_{2}$ batteries: From fundamental chemistry to advanced characterization and effective protection. Batteries Supercaps 2, 638-658. doi: 10.1002/batt.201900031

Hu, Z., Li, G., Wang, A., Luo, J., and Liu, X. (2020). Recent progress of electrolyte design for lithium metal batteries. Batteries Supercaps 3, 331-335. doi: 10.1002/batt.201900191

Hu, Y., Chen, W., Lei, T. Y., Jiao, Y., Wang, H. B., Wang, X. P., et al. (2020). Graphene quantum dots as the nucleation sites and interfacial regulator to suppress lithium dendrites for high-loading lithium-sulfur battery. Nano Energy 68:104373. doi: 10.1016/j.nanoen.2019.104373

Huang, X., Feng, X., Zhang, B., Zhang, L., Zhang, S., Gao, B., et al. (2019). Lithiated $\mathrm{NiCo}_{2} \mathrm{O}_{4}$ nanorods anchored on $3 \mathrm{D}$ nickel foam enable homogeneous $\mathrm{Li}$ plating/stripping for high-power dendrite-free lithium metal anode. ACS Appl. Mater. Inter. 11, 31824-31831. doi: 10.1021/acsami.9b08438

Huang, Y., Chen, B., Duan, J., Yang, F., Wang, T., Wang, Z., et al. (2020). Graphitic carbon nitride $\left(\mathrm{g}-\mathrm{C}_{3} \mathrm{~N}_{4}\right)$ : An interface enabler for solid-state lithium metal batteries. Angew. Chem. Int. Ed. 59, 3699-3704. doi: 10.1002/anie.2019 14417

Huang, Z., Ren, J., Zhang, W., Xie, M., Li, Y., Sun, D., et al. (2018). Protecting the Li-metal anode in a $\mathrm{Li}-\mathrm{O}_{2}$ battery by using boric acid as an SEI-forming additive. Adv. Mater. 30:e1803270. doi: 10.1002/adma.201803270

Huang, K., Li, Z., Xu, Q. J., Liu, H. M., Li, H. X., and Wang, Y. G. (2019). Lithiophilic $\mathrm{CuO}$ nanoflowers on Ti-mesh inducing lithium lateral plating enabling stable lithium-metal anodes with ultrahigh rates and ultralong cycle life. Adv. Energy Mater. 9:1900853. doi: 10.1002/aenm.201900853

Huo, H., Li, X., Sun, Y., Lin, X., Doyle-Davis, K., Liang, J., et al. (2020). $\mathrm{Li}_{2} \mathrm{CO}_{3}$ effects: New insights into polymer/garnet electrolytes for dendrite-free solid lithium batteries. Nano Energy 73:104836. doi: 10.1016/j.nanoen.2020.104836

Jeong, J., Chun, J., Lim, W. G., Kim, W., Jo, C., and Lee, J. (2020). Mesoporous carbon host material for stable lithium metal anode. Nanoscale 12, 11818-11824. doi: 10.1039/d0nr02258f

Jin, S., Jiang, Y., Ji, H., and Yu, Y. (2018). Advanced 3D current collectors for lithium-based batteries. Adv. Mater. 30:e1802014. doi: 10.1002/adma.201802014

Jorge, A. B., Jervis, R., Periasamy, A. P., Qiao, M., Feng, J., Tran, L. N., et al. (2019). $3 \mathrm{D}$ carbon materials for efficient oxygen and hydrogen electrocatalysis. Adv. Energy Mater. 10:1902494. doi: 10.1002/aenm.201902494

Lee, J. I., Song, G., Cho, S., Han, D. Y., and Park, S. (2020). Lithium metal interface modification for high-energy batteries: approaches and characterization. Batter. Supercaps. 3, 828-859. doi: 10.1002/batt.202000016

Li, C., Liu, S., Shi, C., Liang, G., Lu, Z., Fu, R., et al. (2019). Two-dimensional molecular brush-functionalized porous bilayer composite separators toward ultrastable high-current density lithium metal anodes. Nat. Commun. 10:1363. doi: 10.1038/s41467-019-09211-Z

Li, W. T., Zhang, Y. F., Li, H., Chen, Z. J., Shang, T. X., Wu, Z. T., et al. (2020). Layered MXene protected lithium metal anode as an efficient polysulfide blocker for lithium-sulfur batteries. Batteries Supercaps 3, 892-899. doi: 10.1002/batt.202000062

Li, X., Zheng, J. M., Ren, X. D., Engelhard, M. H., Zhao, W. G., Li, Q. Y., et al. (2018). Dendrite-free and performance-enhanced lithium metal batteries through optimizing solvent compositions and adding combinational additives. Adv. Energy Mater. 8:1703022. doi: 10.1002/aenm.201703022

Li, K., Hu, Z., Ma, J., Chen, S., Mu, D., and Zhang, J. (2019). A 3D and stable lithium anode for high-performance lithium-iodine batteries. Adv. Mater. 31:e1902399. doi: 10.1002/adma.201902399
Li, Q., Pan, H., Li, W., Wang, Y., Wang, J., Zheng, J., et al. (2018). Homogeneous interface conductivity for lithium dendrite-free anode. ACS Energy Lett. 3, 2259-2266. doi: 10.1021/acsenergylett.8b01244

Li, S. Y., Liu, Q. L., Zhou, J. J., Pan, T., Gao, L. N., Zhang, W. D., et al. (2019). Hierarchical $\mathrm{Co}_{3} \mathrm{O}_{4}$ nanofiber-carbon sheet skeleton with superior $\mathrm{Na} / \mathrm{Li}$ philic property enabling highly stable alkali metal batteries. Adv. Funct. Mater. 29:1808847. doi: 10.1002/adfm.201808847

Li, Z., Xu, N., Sha, Y., Ji, J., Liu, T., Yan, L., et al. (2020). Chitosan oligosaccharide derived polar host for lithium deposition in lithium metal batteries. Sustain. Mater. Techno. 24:e00158. doi: 10.1016/j.susmat.2020.e00158

Liang, Z., Lin, D., Zhao, J., Lu, Z., Liu, Y., Liu, C., et al. (2016). Composite lithium metal anode by melt infusion of lithium into a $3 \mathrm{D}$ conducting scaffold with lithiophilic coating. Proc. Natl. Acad. Sci. U.S.A. 113, 2862-2867. doi: 10.1073/pnas.1518188113

Liu, B., Wen, J., Chen, H., Yang, M., Liu, Y., and Li, H. (2020). O/N Codoped, layered porous carbon with mesoporosity up to $99 \%$ for ultrahigh-rate capability supercapacitors. Batteries Supercaps. doi: 10.1002/batt.202000037

Liu, H., Liu, X. X., Li, W., Guo, X., Wang, Y., Wang, G. X., et al. (2017). Porous carbon composites for next generation rechargeable lithium batteries. Adv. Energy Mater. 7:1700283. doi: 10.1002/aenm.201700283

Ma, J., Gao, L., Li, S., Zeng, Z., Zhang, L., and Xie, J. (2020). Dual play of chitin-derived N-doped carbon nanosheets enabling high-performance Na$\mathrm{SeS}_{2}$ half/full cells. Batteries Supercaps 3, 165-173. doi: 10.1002/batt.201900159

Maroufi, S., Assefi, M., Nekouei, R. K., and Sahajwalla, V. (2020). Recovery of lithium and cobalt from waste lithium-ion batteries through a selective isolation-suspension approach. Sustain. Mater. Techno. 23:e00139. doi: 10.1016/j.susmat.2019.e00139

Nan, J. X., Guo, X., Xiao, J., Li, X., Chen, W. H., Wu, W. H., et al. (2019). Nanoengineering of 2D MXene-based materials for energy storage applications. Small e1902085. doi: 10.1002/smll.201902085

Pan, J., Tian, X. L., Zaman, S., Dong, Z., Liu, H., Park, H. S., et al. (2019). Recent progress on transition metal oxides as bifunctional catalysts for lithium-air and zinc-air batteries. Batteries Supercaps 2, 336-347. doi: 10.1002/batt.201800082

Pan, L., Wang, J., Luo, Z., Zhao, Z., Li, Y., Egun, I., et al. (2020). 3D periodic ion transport channel to suppress top deposition toward stable lithium metal anode. Batteries Supercaps 3, 773-779. doi: 10.1002/batt.202000001

Park, J., Moon, J., Kim, C., Kang, J. H., Lim, E., Park, J., et al. (2016). Graphene quantum dots: structural integrity and oxygen functional groups for high sulfur/sulfide utilization in lithium sulfur batteries. Npg Asia Mater. 8:e272. doi: 10.1038/am.2016.61

Pei, F., Fu, A., Ye, W., Peng, J., Fang, X., Wang, M. S., et al. (2019). Robust lithium metal anodes realized by lithiophilic $3 \mathrm{D}$ porous current collectors for constructing high-energy lithium-sulfur batteries. ACS Nano 13, 8337-8346. doi: 10.1021/acsnano.9b03784

Pellow, M. A., Ambrose, H., Mulvaney, D., Betita, R., and Shaw, S. (2020). Research gaps in environmental life cycle assessments of lithium ion batteries for gridscale stationary energy storage systems: end-of-life options and other issues. Sustain. Mater. Techno. 23:e0120. doi: 10.1016/j.susmat.2019.e00120

Shi, Q. X., Guan, X., Pei, H. J., Chang, C., Qu, H., Xie, X. L., et al. (2020). Functional covalent triazine frameworks-based quasi-solid-state electrolyte used to enhance lithium metal battery safety. Batteries Supercaps 3, 936-945. doi: 10.1002/batt.202000069

Sloop, S. E., Crandon, L., Allen, M., Lerner, M. M., Zhang, H., Sirisaksoontorn, W., et al. (2019). Cathode healing methods for recycling of lithium-ion batteries. Sustain. Mater. Techno. 22:e00113. doi: 10.1016/j.susmat.2019.e00113

Sun, C., Li, Y., Jin, J., Yang, J., and Wen, Z. (2019). ZnO nanoarray-modified nickel foam as a lithiophilic skeleton to regulate lithium deposition for lithium-metal batteries. J. Mater. Chem. A 7, 7752-7759. doi: 10.1039/c9ta00862d

Tam, T. V., Kang, S. G., Kim, M. H., Lee, S. G., Hur, S. H., Chung, J. S., et al. (2019). Novel graphene hydrogel/B-doped graphene quantum dots composites as trifunctional electrocatalysts for $\mathrm{Zn}$-Air batteries and overall water splitting. Adv. Energy Mater. 9:900945. doi: 10.1002/aenm.201900945

Tang, X., Zhou, D., Li, P., Guo, X., Sun, B., Liu, H., et al. (2020). MXenebased dendrite-free potassium metal batteries. Adv. Mater. 32:e1906739. doi: 10.1002/adma.201906739

Tao, L., Hu, A. Y., Yang, Z. J., Xu, Z. R., Wall, C. E., Esker, A. R., et al. (2020). A surface chemistry approach to tailoring the hydrophilicity and lithiophilicity of 
carbon films for hosting high-performance lithium metal anodes. Adv. Funct. Mater. 30:2000585. doi: 10.1002/adfm.202000585

Tao, R., Bi, X. X., Li, S., Yao, Y., Wu, F., Wang, Q., et al. (2017). Kinetics Tuning the electrochemistry of lithium dendrites formation in lithium batteries through electrolytes. ACS Appl. Mater. Inter. 9, 7003-7008. doi: 10.1021/acsami.6b13859

Tian, H., Zhang, C., Su, P. P., Shen, Z. F., Liu, H., Wang, G. X., et al. (2020). Metal-organic-framework-derived formation of Co-N-doped carbon materials for efficient oxygen reduction reaction. J. Energy Chem. 40, 137-143. doi: 10.1016/j.jechem.2019.03.004

Wang, J., Huang, G., and Zhang, X. B. (2020). Interface between lithium metal and garnet electrolyte: Recent progress and perspective. Batteries Supercaps. doi: 10.1002/batt.202000082

Wang, M. Q., Peng, Z., Luo, W. W., Ren, F. H., Li, Z. D., Zhang, Q., et al. (2019). Tailoring lithium deposition via an SEI-functionalized membrane derived from LiF decorated layered carbon structure. Adv. Energy Mater. 9:1802912. doi: 10.1002/aenm.201802912

Wang, S. H., Yin, Y. X., Zuo, T. T., Dong, W., Li, J. Y., Shi, J. L., et al. (2017). Stable Li metal anodes via regulating lithium plating/stripping in vertically aligned microchannels. Adv. Mater. 29:1703729. doi: 10.1002/adma.201703729

Wei, X. J., Zhang, Y. B., Zhang, B. K., Lin, Z., Wang, X. P., Hu, P., et al. (2019). Yolk-shell-structured zinc-cobalt binary metal sulfide@Ndoped carbon for enhanced lithium-ion storage. Nano Energy 64:103899. doi: 10.1016/j.nanoen.2019.103899

Widijatmoko, S. D., Gu, F., Wang, Z., and Hall, P. (2020). Selective liberation in dry milled spent lithium-ion batteries. Sustain. Mater. Techno. 23:e00134. doi: 10.1016/j.susmat.2019.e00134

Wu, S., Zhang, Z., Lan, M., Yang, S., Cheng, J., Cai, J., et al. (2018). Lithiophilic Cu$\mathrm{CuO}-\mathrm{Ni}$ hybrid structure: Advanced current collectors toward stable lithium metal anodes. Adv. Mater. 30:1705830. doi: 10.1002/adma.201705830

Xiao, J., Zhang, F., Tang, K., Li, X., Wang, D., Wang, Y., et al. (2019). Rational design of a P2-type spherical layered oxide cathode for high-performance sodium-ion batteries. ACS Cent. Sci. 5, 1937-1945. doi: 10.1021 acscentsci.9b00982

Xie, J., Li, B. Q., Song, Y. W., Peng, H. J., and Zhang, Q. (2019). A supramolecular electrolyte for lithium-metal batteries. Batteries Supercaps 3, 47-51. doi: 10.1002/batt.201900112

Xue, P., Sun, C., Li, H., Liang, J., and Lai, C. (2019). Superlithiophilic amorphous $\mathrm{SiO}_{2}-\mathrm{TiO}_{2}$ distributed into porous carbon skeleton enabling uniform lithium deposition for stable lithium metal batteries. Adv. Sci. 6:1900943. doi: 10.1002/advs.201900943

Yan, C., Yao, Y. X., Chen, X., Cheng, X. B., Zhang, X. Q., Huang, J. Q., et al. (2018). Lithium nitrate solvation chemistry in carbonate electrolyte sustains high-voltage lithium metal batteries. Angew. Chem. Int. Ed. 57, 14055-14059. doi: 10.1002/anie.201807034

Yan, J., Dong, K., Zhang, Y., Wang, X., Aboalhassan, A. A., Yu, J., et al. (2019). Multifunctional flexible membranes from sponge-like porous carbon nanofibers with high conductivity. Nat. Commun. 10:5584. doi: 10.1038/s41467-019-13430-9

Yan, J., Liu, F., Hu, Z., Gao, J., Zhou, W., Huo, H., et al. (2020). Realizing dendritefree lithium deposition with a composite separator. Nano Lett. 20, 3798-3807. doi: 10.1021/acs.nanolett.0c00819

Yang, G., Chen, J., Xiao, P., Agboola, P. O., Shakir, I., and Xu, Y. (2018). Graphene anchored on $\mathrm{Cu}$ foam as a lithiophilic 3D current collector for a stable and dendrite-free lithium metal anode. J. Mater. Chem. A 6, 9899-9905. doi: $10.1039 / \mathrm{c} 8 \mathrm{ta}$ 02810a

Yang, G., Li, Y., Tong, Y., Qiu, J., Liu, S., Zhang, S., et al. (2019). Lithium plating and stripping on carbon nanotube sponge. Nano Lett. 19, 494-499. doi: 10.1021/acs.nanolett.8b04376

Yang, T., Liu, Y. G., Yang, D. X., Deng, B. B., Huang, Z. H., Ling, C. D., et al. (2019). Bimetallic metal-organic frameworks derived Ni-Co-Se@C hierarchical bundle-like nanostructures with high-rate pseudocapacitive lithium ion storage. Energy Storage Mater. 17, 374-384. doi: 10.1016/j.ensm.2018.05.024

Ye, H., Zhang, Y., Yin, Y. X., Cao, F. F., and Guo, Y. G. (2020). An outlook on low-volume-change lithium metal anodes for long-life batteries. ACS Cent. Sci. 6, 661-671. doi: 10.1021/acscentsci.0c00351

Yue, X. Y., Bao, J., Yang, S. Y., Luo, R. J., Wang, Q. C., Wu, X. J., et al. (2020). Petaloid-shaped $\mathrm{ZnO}$ coated carbon felt as a controllable host to construct hierarchical Li composite anode. Nano Energy 71:104614. doi: 10.1016/j.nanoen.2020.104614

Yue, X. Y., Wang, W. W., Wang, Q. C., Meng, J. K., Wang, X. X., Song, Y., et al. (2019b). Cuprite-coated $\mathrm{Cu}$ foam skeleton host enabling lateral growth of lithium dendrites for advanced Li metal batteries. Energy Storage Mater. 21, 180-189. doi: 10.1016/j.ensm.2018.12.007

Yue, X. Y., Li, X. L., Wang, W. W., Chen, D., Qiu, Q. Q., Wang, Q. C., et al. (2019a). Wettable carbon felt framework for high loading Li-metal composite anode. Nano Energy 60, 257-266. doi: 10.1016/j.nanoen.2019.03.057

Zhang, Q., Liu, S., Lin, Z., Wang, K., Chen, M., Xu, K., et al. (2020). Highly safe and cyclable Li-metal batteries with vinylethylene carbonate electrolyte. Nano Energy 74:104860. doi: 10.1016/j.nanoen.2020.104860

Zhang, R., Li, N. W., Cheng, X. B., Yin, Y. X., Zhang, Q., and Guo, Y. G. (2017). Advanced micro/nanostructures for lithium metal anodes. Adv. Sci. 4:1600445. doi: $10.1002 /$ advs. 201600445

Zhang, T., Han, S., Guo, W., Hou, F., Liu, J., Yan, X., et al. (2019). Continuous carbon nanotube composite fibers for flexible aqueous lithium-ion batteries. Sustain. Mater. Techno. 20:e00096. doi: 10.1016/j.susmat.2019.e00096

Zhang, X. Q., Cheng, X. B., Chen, X., Yan, C., and Zhang, Q. (2017). Fluoroethylene carbonate additives to render uniform $\mathrm{Li}$ deposits in lithium metal batteries. Adv. Funct. Mater. 27:1605989. doi: 10.1002/adfm.201605989

Zhang, Z., Sun, J., Wang, F., and Dai, L. (2018a). Efficient oxygen reduction reaction (ORR) catalysts based on single iron atoms dispersed on a hierarchically structured porous carbon framework. Angew. Chem. Int. Ed. 57, 9038-9043. doi: 10.1002/anie.201804958

Zhang, C., Lv, W., Zhou, G. M., Huang, Z. J., Zhang, Y. B., Lyu, R. Y., et al. (2018). Vertically aligned lithiophilic $\mathrm{CuO}$ nanosheets on a $\mathrm{Cu}$ collector to stabilize lithium deposition for lithium metal batteries. Adv. Energy Mater. 8:1703404. doi: 10.1002/aenm.201703404

Zhang, D. M., Chen, Z. W., Bai, J., Yang, C. C., and Jiang, Q. (2020). Highly nitrogen-doped porous carbon nanosheets as high-performance anode for potassium-ion batteries. Batteries Supercaps 3, 185-193. doi: 10.1002/batt.201900144

Zhang, R., Chen, X., Shen, X., Zhang, X. Q., Chen, X. R., Cheng, X. B., et al. (2018a). Coralloid carbon fiber-based composite lithium anode for robust lithium metal batteries. Joule 2, 764-777. doi: 10.1016/j.joule.2018.02.001

Zhang, R., Wen, S. W., Wang, N., Qin, K. Q., Liu, E. Z., Shi, C. S., et al. (2018b). $\mathrm{N}$-doped graphene modified $3 \mathrm{D}$ porous $\mathrm{Cu}$ current collector toward microscale homogeneous Li deposition for Li metal anodes. Adv. Energy Mater. 8:1800914. doi: 10.1002/aenm.201800914

Zhang, S. J., You, J. H., Chen, J. D., Hu, Y. Y., Wang, C. W., Liu, Q., et al. (2019). Aluminum-based metal-organic frameworks derived $\mathrm{Al}_{2} \mathrm{O}_{3}$-loading mesoporous carbon as a host matrix for lithium-metal anodes. ACS Appl. Mater. Inter. 11, 47939-47947. doi: 10.1021/acsami.9b16363

Zhang, W., Fu, Y., Liu, W., Lim, L., Wang, X., and Yu, A. (2019). A general approach for fabricating $3 \mathrm{D} \mathrm{MFe} \mathrm{O}_{4}(\mathrm{M}=\mathrm{Mn}, \mathrm{Ni}, \mathrm{Cu}, \mathrm{Co}) /$ graphitic carbon nitride covalently functionalized nitrogen-doped graphene nanocomposites as advanced anodes for lithium-ion batteries. Nano Energy 57, 48-56. doi: 10.1016/j.nanoen.2018.12.005

Zhang, W., Xu, T., Liu, Z., Wu, N. L., and Wei, M. (2018). Hierarchical $\mathrm{TiO}_{2-x}$ imbedded with graphene quantum dots for high-performance lithium storage. Chem. Commun. 54, 1413-1416. doi: 10.1039/C7CC09406J

Zhang, X., Fan, Y., Khan, M. A., Zhao, H., Ye, D., Wang, J., et al. (2020). Co-Ni binary-metal oxide coated with porous carbon derived from metalorganic framework as host of nano-sulfur for lithium-sulfur batteries. Batteries Supercaps 3, 108-116. doi: 10.1002/batt.201900121

Zhang, Y., Shi, Y., Hu, X. C., Wang, W. P., Wen, R., Xin, S., et al. (2019). A 3D lithium/carbon fiber anode with sustained electrolyte contact for solid-state batteries. Adv. Energy Mater. 10:1903325. doi: 10.1002/aenm.2019 03325

Zhang, Y., Wang, C. W., Pastel, G., Kuang, Y. D., Xie, H., Li, Y. J., et al. (2018). 3D wettable framework for dendrite-free alkali metal anodes. Adv. Energy Mater. 8:1800635. doi: 10.1002/aenm.201800635

Zhang, Z., Wang, Z. L., and Lu, X. (2018b). Multishelled Si@Cu microparticles supported on 3D $\mathrm{Cu}$ current collectors for stable and binder-free anodes of lithium-ion batteries. ACS Nano 12, 3587-3599. doi: 10.1021/acsnano. $8 \mathrm{~b} 00703$ 
Zhao, F., Zhou, X. F., Deng, W., and Liu, Z. P. (2019). Entrapping lithium deposition in lithiophilic reservoir constructed by vertically aligned $\mathrm{ZnO}$ nanosheets for dendrite-free Li metal anodes. Nano Energy 62, 55-63. doi: 10.1016/j.nanoen.2019.04.087

Zhou, T., Shen, J. D., Wang, Z. S., Liu, J., Hu, R. Z., Ouyang, L. Z., et al. (2020). Regulating lithium nucleation and deposition via MOF-derived Co@Cmodified carbon cloth for stable Li metal anode. Adv. Funct. Mater. 30, 1909159. doi: 10.1002/adfm.201909159

Zhou, Y., Doerrer, C., Kasemchainan, J., Bruce, P. G., Pasta, M., and Hardwick, L. J. (2020). Observation of interfacial degradation of $\mathrm{Li}_{6} \mathrm{PS}_{5} \mathrm{Cl}$ against lithium metal and $\mathrm{LiCoO}_{2}$ via in situ electrochemical raman microscopy. Batteries Supercaps 3, 647-652. doi: 10.1002/batt.201900218

Zhou, Z. L., Li, N., Yang, Y. Z., Chen, H. S., Jiao, S. Q., Song, W. L., et al. (2018). Ultra-lightweight 3D carbon current collectors: constructing all-carbon electrodes for stable and high energy density dual-ion batteries. Adv. Energy Mater. 8:1801439. doi: 10.1002/aenm.201801439

Zhu, J. G., Li, P. K., Chen, X., Legut, D., Fan, Y. C., Zhang, R. F., et al. (2019). Rational design of graphitic-inorganic Bi-layer artificial
SEI for stable lithium metal anode. Energy Storage Mater. 16, 426-433. doi: 10.1016/j.ensm.2018.06.023

Zuo, T. T., Yin, Y. X., Wang, S. H., Wang, P. F., Yang, X., Liu, J., et al. (2018). Trapping lithium into hollow silica microspheres with a carbon nanotube core for dendrite-free lithium metal anodes. Nano Lett. 18, 297-301. doi: 10.1021/acs.nanolett.7b04136

Conflict of Interest: The authors declare that the research was conducted in the absence of any commercial or financial relationships that could be construed as a potential conflict of interest.

Copyright (c) 2020 Tang, Xiao, Li, Wang, Long, Chen, Gao, Chen, Liu and Liu. This is an open-access article distributed under the terms of the Creative Commons Attribution License (CC BY). The use, distribution or reproduction in other forums is permitted, provided the original author(s) and the copyright owner(s) are credited and that the original publication in this journal is cited, in accordance with accepted academic practice. No use, distribution or reproduction is permitted which does not comply with these terms. 